\title{
EFFETS A LONG TERME DES FUMURES MINERALES ET ORGANO-MINERALES SUR LA DYNAMIQUE DU PHOSPHORE DANS UN LIXISOL AU BURKINA FASO
}

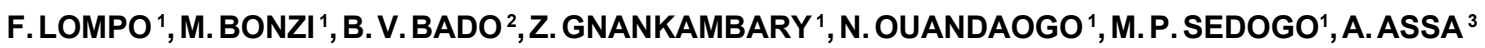 \\ ${ }^{1}$ Institut de l'Environnement et de Recherches Agricoles (INERA), 04 BP 8645 Ouagadougou 04, Burkina Faso. \\ E-mail : frlompo@yahoo.fr \\ ${ }^{2}$ Centre du Riz pour l'Afrique (ADRAO), BP 96 Saint Louis, Sénégal ; \\ ${ }^{3}$ Unité de Formation et de Recherches en Sciences de la Terre et des Ressources Minières \\ Université de Cocody, 22 BP 582 Abidjan 22, Côte d'Ivoire.
}

\begin{abstract}
RESUME
La disponibilité et la cinétique du phosphore $(P)$ dans un Lixisol, après 40 années de mise en culture, ont été étudiées par la méthode de la dilution isotopique. Quatre traitements ont été utilisés : fumures minérales faible (fm) et forte (FM), organo-minérale faible (fmo) et le témoin $\left(T_{1}\right)$. Les fumures ont eu des effets sur la cinétique de $\mathrm{P}$ du sol. Les fumures minérales ont influencé le Facteur Intensité ( $\mathrm{Cp}$ ). Comparativement à $\mathrm{T}_{1}\left(\mathrm{Cp}=0,018 \mathrm{mg} \mathrm{PL}^{-1}\right)$, ce facteur a été de 0,$038 ; 0,053$ et $0,177 \mathrm{mg} \mathrm{PL}^{-1}$ pour fm, FM et fmo, respectivement. Le phosphore isotopiquement échangeable $\left(E_{1}\right)$ a été également amélioré $(2,44 ; 1,69 ; 3,20$; et $0,96 \mathrm{mg}$ $P L^{-1}$ pour $\mathrm{fm}, \mathrm{FM}$, fmo et $T_{1}$, respectivement). Seule fm a augmenté le Facteur Capacité $\left(E_{1} / C p\right)$ qui a été de 53,$33 ; 64,21 ; 31,89$ et $18,08 \mathrm{~L} \mathrm{~kg}^{-1}$ respectivement pour $\mathrm{T}_{1}, \mathrm{fm}, \mathrm{FM}$ et fmo. La fumure organo-minérale faible a diminué la fixation de $\mathrm{P}(49 \%)$, alors que les fumures minérales l'ont augmentée de 69 à $84 \%$.
\end{abstract}

Mots clés : Disponibilité, phosphore, cinétique, Lixisol, dilution isotopique.

\begin{abstract}
LONG-TERM EFFECT OF MINERAL AND ORGANO-MINERAL FERTILIZERS ON PHOSPHORUS DYNAMIC IN A LIXISOL OF BURKINA FASO.
\end{abstract}

The availability and kinetic of phosphorous $(P)$ in a Lixisol were studied with soil samples from a long-term soil fertility experiment (40 years) in Burkina Faso using isotopic dilution method. Four treatments were used: low rate of mineral fertilizer ( $\mathrm{fm}$ ), high rate of mineral fertilizer (FM), low rate of organo-mineral fertilizer (fmo) and a control $\left(T_{1}\right)$. The results show that soil $P$ kinetics were affected by fertilizers. The application of mineral fertilizer increased the intensity factor $(C p)$. Compared to the control ( $\left.C p=0.018 \mathrm{mg} \mathrm{PL}^{-1}\right)$, the intensity factors with $\mathrm{fm}, \mathrm{FM}$ and fmo were 0.038; 0.053 and $0.177 \mathrm{mg} \mathrm{P} \mathrm{L}^{-1}$, respectively. They also improved exchangeable

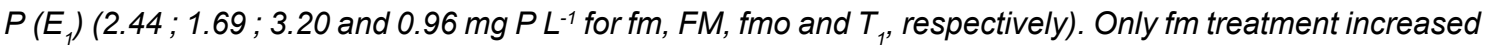
the capacity factors $\left(E_{1} / C p\right)$, which were 53.33; $64.21 ; 31.89$ and $18.08 \mathrm{~L} \mathrm{~kg}^{-1}$ for $T_{1}, \mathrm{fm}$, FM and fmo, respectively. Mineral fertilizers increased $P$ fixation from $69 \%$ (control) to $84 \%$, while organo-mineral fertilizer decreased it (49\%).

Key-words : Availability, phosphorus, kinetics, Lixisol, isotopic exchange.

\section{INTRODUCTION}

Les plantes s'alimentent en éléments nutritifs à partir principalement de la solution du sol, à travers leurs racines. C'est généralement à travers un processus d'adsorption/désorption entre les phases solide et liquide du sol que les éléments nutritifs sont rendus plus ou moins accessibles à la plante (Frossard et Sinaj, 1997). Pour le phosphore, la forme dite assimilable a pendant longtemps été évaluée par des extractions chimiques, présentant toutes des avantages et des inconvénients (Calvet, 1988 ; Boniface et Trocmé, 1988). En particulier, 
les méthodes d'extraction chimique ne permettent pas de prédire les productions des plantes dans différents environnements (Bationo et al.,1991). Depuis les travaux de Beckett et White (1964) qui ont proposé de caractériser la biodisponibilité du phosphore par les 3 facteurs Intensité, Quantité et Capacité, des auteurs, dont Fardeau et al. (1991) ont mis au point des méthodes permettant de prendre en compte les vitesses de renouvellement des ions phosphates de la solution du sol à partir de sa phase solide dans l'évaluation de la nutrition phosphatée des plantes. Le facteur Intensité correspond à la concentration des ions phosphates dans la solution du sol, le facteur Quantité est la réserve d'ions phosphates en mesure d'alimenter la solution du sol $\mathrm{P}$ pour les besoins des plantes, et enfin le facteur Capacité correspond à l'aptitude du sol à maintenir constant le facteur Intensité lorsque le facteur Quantité fluctue. En plus de ces trois facteurs, des constantes descriptives des mouvements potentiels des ions phosphates entre les phases liquide et solide du sol ont été définies. La constante $\mathrm{Km}$ $\left(\mathrm{mn}^{-1}\right)$ caractérise la vitesse de transfert des ions phosphates ; la constante $\mathrm{Tm}(\mathrm{mn})$ représente le temps moyen de séjour des ions dans la phase liquide et enfin la constante $\mathrm{Fm}$ (mg P mn-1 $\mathrm{kg}^{-1}$ ), exprime le flux moyen d'échange et de transfert des ions phosphates entre les deux phases.

En Afrique de l'Ouest sahélienne, les techniques isotopiques ont été utilisées pour étudier l'impact des engrais phosphatés sur la nutrition des plantes et sur le statut du phosphore dans les sols. Fardeau et Jappe (1980) ont montré que la cinétique de dilution isotopique peut permettre d'évaluer la rentabilité agronomique d'une fertilisation de redressement. Sinaj et al. (2001) en comparant les effets de différentes stratégies de gestion de la fertilité des sols sur la biodisponibilité du phosphore dans 4 types de sols de l'Afrique de l'Ouest, dont deux de la zone sahélienne, ont montré que : (i) le mulch avec des résidus de récolte en association avec des engrais phosphatés solubles permettait non seulement d'améliorer la nutrition phosphatée des plantes, mais contribuait également à accroître de façon durable les rendements ; (ii) l'application à la volée du phosphate super simple (SSP) à la dose de $13 \mathrm{~kg} \mathrm{P} \mathrm{ha-1}$ permettait d'accroître les facteurs Intensité et Quantité ; et (iii) l'application localisée de $4 \mathrm{~kg} \mathrm{ha}^{-1}$ conduisait à une amélioration du facteur Quantité et de l'efficience agronomique du SSP. Au Burkina Faso, Compaoré et al. (2003), à travers une étude sur l'influence de modes d'utilisation des terres sur le phosphore isotopiquement échangeable, ont d'une part confirmé le fait que le phosphore est l'un des principaux facteurs limitants de la production agricole et, d'autre part, montré que l'apport d'engrais phosphatés était l'unique moyen de lever ladite contrainte et d'améliorer le bilan en phosphore des sols.

Rares sont les études qui se sont intéressées cependant aux effets à long terme de fumures minérales et organo-minérales sur les paramètres caractéristiques de la cinétique de transfert des ions phosphates et sur les constantes de vitesse d'échange.

L'objectif de la présente étude est d'évaluer les effets de 40 années de fumures minérales (faible et forte) et de la fumure organo-minérale sur certains paramètres de mobilité du phosphore entre les phases solide et liquide du sol, en vue de mieux comprendre la nutrition phosphatée du sorgho.

\section{MATERIEL ET METHODES}

\section{CARACTERISTIQUES GENERALES DU SITE D'ETUDE}

Situé dans la zone Nord- Soudanienne (Fontes et Guinko, 1995), à $300 \mathrm{~m}$ d'altitude, $12^{\circ} 16^{\prime} \mathrm{N}$, $2^{\circ} 9^{\prime} \mathrm{O}$, le site de Saria est caractérisé par une pluviométrie irrégulière aussi bien dans l'espace que dans le temps (entre mai et octobre). La moyenne annuelle pluviométrique des 30 dernières années est de $800 \mathrm{~mm}$.

Le dispositif est installé sur un Lixisol (luss et al., 2006) dont les caractéristiques à la mise en place de l'étude en 1960 (Tableau 1) ont révélé un sol sableux en surface $(0-9 \mathrm{~cm})$ à argileux dans I'horizon $9-25 \mathrm{~cm}$, riche en matière organique, pauvre en phosphore total et assimilable ainsi qu'en azote. Le complexe absorbant est moyennement saturé, avec une somme de bases échangeables et une CEC faibles. Les teneurs en $\mathrm{Ca}^{2+}, \mathrm{Mg}^{2+}$ et $\mathrm{K}^{+}$ont été respectivement faibles, très faibles et moyennes. Ce sol est moyennement acide ( $\mathrm{pH}$ eau $=5,90)$. Le type d'argile dominant est la kaolinite $(63 \%)$. 
Tableau 1 : Caractéristiques physico-chimiques initiales du sol étudié.

Initial soil physical and chemical characteristics.

\begin{tabular}{|c|c|c|c|c|}
\hline & \multirow{2}{*}{ Caractéristiques physico-chimiques } & \multicolumn{2}{|c|}{ Horizon } & \multirow{2}{*}{$\begin{array}{c}\text { Moyenne } \\
0-25 \mathrm{~cm}\end{array}$} \\
\hline & & $0-9 \mathrm{~cm}$ & $9-25 \mathrm{~cm}$ & \\
\hline \multirow{4}{*}{ Granulométrie (\%) } & Argile & 9,0 & 38,3 & 23,7 \\
\hline & Limons & 6,3 & 7,3 & 6,8 \\
\hline & Sables fins & 26,9 & 23,0 & 25,0 \\
\hline & Sables grossiers & 30,5 & 15,3 & 22,9 \\
\hline \multirow{3}{*}{ Matières organiques } & Carbone total $\left(\mathrm{g} \mathrm{kg}^{-1}\right)$ & 5,60 & 6,80 & 6,20 \\
\hline & Azote total $\left(\mathrm{g} \mathrm{kg}^{-1}\right)$ & 0,30 & 0,40 & 0,35 \\
\hline & $\mathrm{C} / \mathrm{N}$ & 18,7 & 17,0 & 17,7 \\
\hline \multirow{2}{*}{ Phosphore (mg P kg-1) } & $\mathrm{P}$ total & 30 & 42 & 36 \\
\hline & $\mathrm{P}$ assimilable & 28 & 24 & 26 \\
\hline \multirow{6}{*}{ Complexe absorbant (cmol kg-1) } & $\mathrm{Ca}^{2+}$ & 1,00 & 2,00 & 1,50 \\
\hline & $\mathrm{Mg}^{2+}$ & 0,30 & 0,20 & 0,25 \\
\hline & $\mathrm{K}^{+}$ & 0,04 & 0,10 & 0,07 \\
\hline & Somme des Bases (S) & 1,70 & 2,60 & 2,15 \\
\hline & Capacité d'échange (T) & 2,20 & 5,10 & 3,65 \\
\hline & Saturation $\mathrm{V}=\mathrm{S} / \mathrm{T}$ en $\%$ & 77 & 51 & 64 \\
\hline \multirow{2}{*}{$\mathrm{pH}$} & $\mathrm{pH}$ eau & 6,4 & 5,3 & 5,9 \\
\hline & $\mathrm{pH} \mathrm{KCl}$ & 5,3 & 4,4 & 4,9 \\
\hline \multirow{3}{*}{ Fer $\left(\mathrm{g} \mathrm{kg}^{-1}\right)$} & Fer libre & 55 & 113 & 84 \\
\hline & Fer Total & 132 & 245 & 189 \\
\hline & Fer libre/fer total $(\%)$ & 42 & 46 & 44 \\
\hline
\end{tabular}

\section{DISPOSITIF EXPERIMENTAL}

L'étude a été réalisée sur des échantillons de sol prélevés dans une expérimentation de longue durée (Essai Entretien de la Fertilité), installée depuis 40 années sur la station de recherche agronomique de Saria au Burkina Faso.

Le dispositif expérimental est un factoriel $6 \times 3$, selon un arrangement en Split-plot, dont les facteurs principaux sont :

$\mathrm{T}_{1}:$ Témoin (sans apport d'engrais)

$T_{2}$ : fmr (fumure minérale faible [14 - 23 - 14] + résidus de sorgho)

$T_{3}$ : fmo (fumure minérale faible [14 - $\left.23-14\right]+$ $5 \mathrm{t}$ de fumier par ha tous les 2 ans)

$\mathrm{T}_{4}$ : fm (fumure minérale faible [14 - 23 - 14])

$T_{5}$ : $F M O$ (fumure minérale forte $[60-23-44]+$ $40 \mathrm{t}$ de fumier par ha tous les 2 ans)

$T_{6}: F M$ (fumure minérale forte [60 - 23 - 44]).

Le fumier produit à Saria a les caractéristiques chimiques suivantes en $\mathrm{g} \mathrm{kg}^{-1}$ (Lompo, 2008) : $\mathrm{N}: 14,9 ; \mathrm{P}: 2,1 ; \mathrm{K}: 23,5 ; \mathrm{Ca}: 10,8$; Mg : 4,9; C : 226,2. Son du C/N est de 15.
Les trois facteurs secondaires ont été : (i) culture continue de sorgho, (ii) rotation sorgho / niébé et, (iii) rotation sorgho / coton.

\section{ECHANTILLONNAGE DE SOLS}

Les sols utilisés proviennent d'échantillons composites qui ont été obtenus à partir de 5 points de prélèvement dans l'horizon $0-25 \mathrm{~cm}$. Ces prélèvements ont été effectués, en saison sèche, en mars 2000, sur les parcelles en culture continue de sorgho ayant reçu les traitements $T_{1}, \mathrm{fmo}, \mathrm{fm}$ et $F M$ pendant 40 ans. Les échantillons ont été séchés à l'air libre et tamisés à $2 \mathrm{~mm}$ avant analyses.

\section{ANALYSES DE SOLS}

Les analyses de sol ont été réalisées, selon les procédures décrites par Walinga et al.(1989), dans les laboratoires de sols et végétaux du Centre de Recherches Environnementales, Agricoles et de Formation de Kamboinsé (Burkina Faso) et du Centre de Coopération Internationale en Recherche Agronomique pour le Développement (CIRAD) à Montpellier, en France. 
La granulométrie a été déterminée par la méthode internationale à la pipette Robinson. Le $\mathrm{pH}$ a été mesuré par la méthode électrométrique avec des rapports sol-solution de 1:2,5. Le carbone total du sol a été déterminée par la méthode Walkley-Black (1934). Pour la détermination des éléments totaux $(N, P, K, C a$ et $\mathrm{Mg}$ ) les échantillons de sol ont été minéralisés par un mélange $\mathrm{H}_{2} \mathrm{SO}_{4}-\mathrm{Se}-\mathrm{H}_{2} \mathrm{O}_{2}$ à $450{ }^{\circ} \mathrm{C}$ pendant $4 \mathrm{~h}$. Puis, les teneurs en $\mathrm{N}$ et $\mathrm{P}$ ont été déterminées par colorimétrie tandis que les teneurs en $\mathrm{K}, \mathrm{Ca}$ et $\mathrm{Mg}$ ont été déterminées à l'aide d'un spectrophotomètre d'absorption atomique à flamme. Les bases échangeables et la CEC ont été déterminées par la méthode au cobaltyhexamine (Fallavier et al., 1985). Le phosphore assimilable a été déterminé selon les méthodes Bray 1 (Bray et Kurtz, 1945) et Olsen (Olsen et al., 1954). La méthode de dilution isotopique (Fardeau, 1996 ; Di et al., 1997 : Frossard et Sinaj, 1997) a été utilisée pour la détermination des paramètres de disponibilité du phosphore des sols ayant reçu les différentes fumures. Cette méthode consiste à agiter $10 \mathrm{~g}$ de sol dans $99 \mathrm{ml}$ d'eau distillée pendant $17 \mathrm{~h}$ pour obtenir un état stationnaire du système solsolution (la concentration des ions phosphates en solution est alors constante). Tout en continuant l'agitation, on injecte $1 \mathrm{ml}$ de solution contenant une quantité connue $\mathrm{R}$ de radioactivité fournie par ${ }^{32} \mathrm{PO}_{4}$. A des temps $t(1,10$ et $40 \mathrm{mn}$ ) des prélèvements de $10 \mathrm{ml}$ de la solution sont effectués et filtrés (filtres de 0,2 $\mu \mathrm{m}$ pour les deux premiers prélèvements et de $0,025 \mu \mathrm{m}$ pour le dernier). La quantité de radioactivité restante $r(t)$ en solution à l'instant $t$, qui est mesurée à l'aide d'un compteur à scintillation liquide, varie avec le temps selon l'équation suivante (Fardeau et al., 1985) :

$r(t) / R=\{r(1) / R\} \times\left\{t+[r(1) / R]^{1 / n}\right\}^{-n}+r(\infty) / R$

où $R$ est la quantité totale de radioactivité introduite ; $r(1)$ et $r(\infty)$ sont, respectivement, la radioactivité restant dans la solution après une minute et l'infini ; $n$ est un paramètre qui décrit le taux de disparition de l'isotope de la solution du sol après une minute.

La quantité $E$ (t) (en $\mathrm{mg} \mathrm{P} \mathrm{kg}^{-1}$ ) du $P$ isotopiquement échangeable, à un instant $t$, est calculée à partir de l'équation suivante :

$$
r(t) /(10 \times C p)=R / E(t)
$$

d'où l'on tire :

$$
E(t)=10 \times C p R / r(t)
$$

où $\mathrm{Cp}$ (Facteur Intensité) est le $\mathrm{P}$ soluble en $\mathrm{mg}$ $\mathrm{P} \mathrm{L}^{-1}$. Le facteur 10 provient du ratio sol $(1 \mathrm{~g})$ : eau $(10 \mathrm{ml})$. La valeur $10 \times C p$ représente le $P$ soluble $\left(\mathrm{mg} \mathrm{kg}^{-1}\right)$ dans l'eau.

Les autres variables descriptives de l'état du $P$ disponible dans le sol ont été calculées de la façon suivante :

- le pouvoir fixateur du sol vis-à-vis de $\mathrm{PO}_{4}{ }^{3-}$ est $r_{1} / R$. Il est élevé si $r_{1} / R$ est inférieur à 0,2 et faible si le rapport est supérieur à 0,8 ;

- la pente (n) de la droite caractérise la cinétique de transfert de $\mathrm{PO}_{4}{ }^{3-}$ entre les phases liquide et solide. Selon Compaoré (1996), ce paramètre varie entre 0 (pour les sols riches en $P$ biodisponible ou pouvant valoriser une fertilisation phosphatée) et 0,5 (pour les sols capables de fixer le phosphore apporté) ;

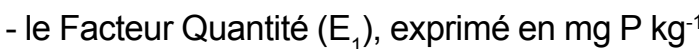
de sol, représente la quantité de $P$ échangée en une minute qui peut être immédiatement assimilée par les racines. E1 varie entre 0,2 et $60 \mathrm{mg} \mathrm{P} \mathrm{kg}^{-1}$ sol dans les sols cultivés. Un sol est considéré comme ne présentant pas de contrainte de nutrition phosphatée si $E_{1}>5 \mathrm{mg}$ $\mathrm{P} \mathrm{kg}^{-1}$ (Fardeau, 1981) ;

- le Facteur Capacité $\left(E_{1} / C p\right)$, exprimé en $\mathrm{L} \mathrm{kg}^{-1} \mathrm{de}$ sol, est le volume apparent de solution de sol présent dans la phase solide avec lequel pourrait être mélangé le $P$ sans que la concentration $\mathrm{Cp}$ des ions varie. Il est généralement compris entre 10 et $2000 \mathrm{~L} \mathrm{~kg}^{-1}$ dans les sols cultivés.

Les variables descriptives des mouvements potentiels de $\mathrm{PO}_{4}{ }^{3-}$ ont été également déterminées :

- la constante moyenne de vitesse de transfert des ions entre les phases solide et liquide $(\mathrm{Km})$, exprimée en $\mathrm{mn}^{-1} ; \mathrm{Km}=\mathrm{n} /\left(\mathrm{r}_{1} / \mathrm{R}\right) 1 / \mathrm{n}$;

- le temps moyen de séjour de $\mathrm{PO}_{4}{ }^{3-}$ dans la phase liquide du sol $(\mathrm{Tm})$, exprimé en $\mathrm{mn}$; $\mathrm{Tm}=1 / \mathrm{Km}$ :

- le flux moyen d'échange de $\mathrm{PO}_{4}^{3-}$ entre les phases solide et liquide du sol $(\mathrm{Fm}) ; \mathrm{Fm}=$ $10 \mathrm{Cp} \times \mathrm{Km}$. Des valeurs de Fm supérieurs à $30 \mathrm{mg} \mathrm{mn}^{-1} \mathrm{~kg}^{-1}$ sont nécessaires pour une nutrition phosphatée satisfaisante (Fardeau, 1981). 


\section{RESULTATS}

\section{EFFETS DES ENGRAIS MINERAUX SUR LA CINETIQUE DE TRANSFERT DES IONS PHOSPHATES D'UN LIXISOL.}

\section{Paramètres de la cinétique de transfert}

La concentration de $\mathrm{P}$ dans la solution du sol (Tableau 2), représentée par le Facteur Intensité $(\mathrm{Cp})$, a été faible pour le témoin absolu $(0,018$ $\mathrm{mg} \mathrm{P} \mathrm{L}^{-1}$ ). Les différents engrais minéraux ont permis d'améliorer, de façon significative, la concentration en $\mathrm{PO}_{4}^{3-}$. Avec la fumure minérale faible, $\mathrm{Cp}$ a été de $0,038 \mathrm{mg} \mathrm{P} \mathrm{L}^{-1}$ soit une augmentation de $111 \%$ par rapport au témoin. Avec la fumure minérale forte, cette augmentation a été de $194 \%$.

Le phosphore isotopiquement échangeable en $1 \mathrm{mn}\left(\mathrm{E}_{1}\right)$ a été surtout influencé par la fumure minérale faible. Comparativement au témoin $\left(E_{1}\right.$ $=0,96 \mathrm{mg} \mathrm{P} \mathrm{L}^{-1}$ ), l'augmentation de ce Facteur Quantité due à fm $\left(E_{1}=2,44 \mathrm{mg} \mathrm{P} \mathrm{L}^{-1}\right)$ a été de $154 \%$. Cette augmentation a été plus faible $(76 \%)$ avec FM $\left(E_{1}=1,69 \mathrm{mg} \mathrm{P} \mathrm{L}^{-1}\right)$.

De façon générale et comparativement au témoin, seule la fumure minérale faible a eu un effet sur le Facteur Capacité $\left(E_{1} / C p\right)$. En effet, les valeurs moyennes de ce facteur ont été de $53,33 \mathrm{~L} \mathrm{~kg}^{-1}$ pour le témoin, de 64,21 et $31,89 \mathrm{~L} \mathrm{~kg}^{-1}$ pour les parcelles ayant reçu les fumures minérales faible et forte, respectivement.

Les valeurs du pouvoir fixateur du sol, vis-à-vis du phosphore, représentées par les rapports $r_{1} / R$, ont été inférieures à 0,2 pour le témoin absolu $(0,187)$ et pour $\mathrm{fm}(0,156)$, mais, plus élevée avec FM $(0,314)$. Comme on peut le constater sur la figure 1 , le pourcentage de $P$ fixé, comparati-vement au $\mathrm{P}$ en solution, a été plus faible (69 \%) sur les parcelles ayant reçu la fumure minérale forte que sur le témoin $(81 \%)$ et les parcelles avec fumure minérale faible $(84 \%)$.

Le pouvoir tampon du sol, vis-à-vis de $\mathrm{P}$, représenté par $R / r_{1}$, a été plus élevé avec fm et $\mathrm{T}_{1}$ (6,4 et 5,3 respectivement), qu'avec la FM $(3,2)$.

Comparativement au témoin, les fumures minérales ont provoqué une baisse du paramètre n. Ces valeurs sont en effet passées de 0,398 pour $T_{1}$ à 0,247 et 0,201 pour fm et $F M$, respectivement.

\section{Constantes moyennes de vitesse d'échange du phosphore}

La constante moyenne de vitesse de transfert des ions entre les phases solide et liquide $(\mathrm{Km})$ a varié significativement en fonction des traitements. Comparativement au témoin, les niveaux de fumure minérale ont augmenté cette constante. L'augmentation a été importante avec $\mathrm{fm}$. Les temps moyens de séjour des ions phosphates dans la phase liquide du sol ( $\mathrm{Tm}$ ) ont été courts $\left(2.10^{-3}\right.$ et $2.10^{-2} \mathrm{mn}$, respectivement pour les fumures minérales faible et forte). Les variations des flux moyens de transfert des ions de la phase solide vers la phase liquide $(\mathrm{Fm})$ montrent un effet très significatif du niveau de fumure minérale. Le flux le plus important a été obtenu avec la fumure minérale faible.

EFFETS DE LA FUMURE ORGANOMINERALE SUR LA CINETIQUE DE TRANSFERT DU PHOSPHORE

\section{Cinétique de transfert du phosphore}

Les effets de la fumure organo-minérale (fmo) sur la cinétique de transfert du phosphore ont été assez différents de ceux obtenus sur les parcelles où la fumure minérale faible seule a été appliquée (Tableau 3). Le Facteur Intensité (Cp) a connu une hausse sensible, de l'ordre de $366 \%$. Les valeurs de $\mathrm{Cp}$ ont été supérieures au seuil de $0,02 \mathrm{mg} \mathrm{P} \mathrm{L}^{-1}$ et montrent que le $P$ n'est pas limitant sur les parcelles ayant reçu fmo. Le Facteur quantité $\left(E_{1}\right)$ a connu également un accroissement, mais moins sensible. Il a été de $31 \%$. Par contre, le Facteur Capacité $\left(E_{1} / C p\right)$ et le pouvoir fixateur du sol $\left(r_{1} / R\right)$, ont connu une baisse. Le pouvoir fixateur a été largement supérieur à 0,2 indiquant ainsi une baisse de la capacité de fixation du phosphore sur les parcelles recevant fmo (Figure 2). Le $P$ fixé, comparativement au phosphore en solution, a été de $45 \%$ avec fmo contre $81 \%$ et $84 \%$ avec $\mathrm{T}_{1}$ et $\mathrm{fm}$, respectivement. 


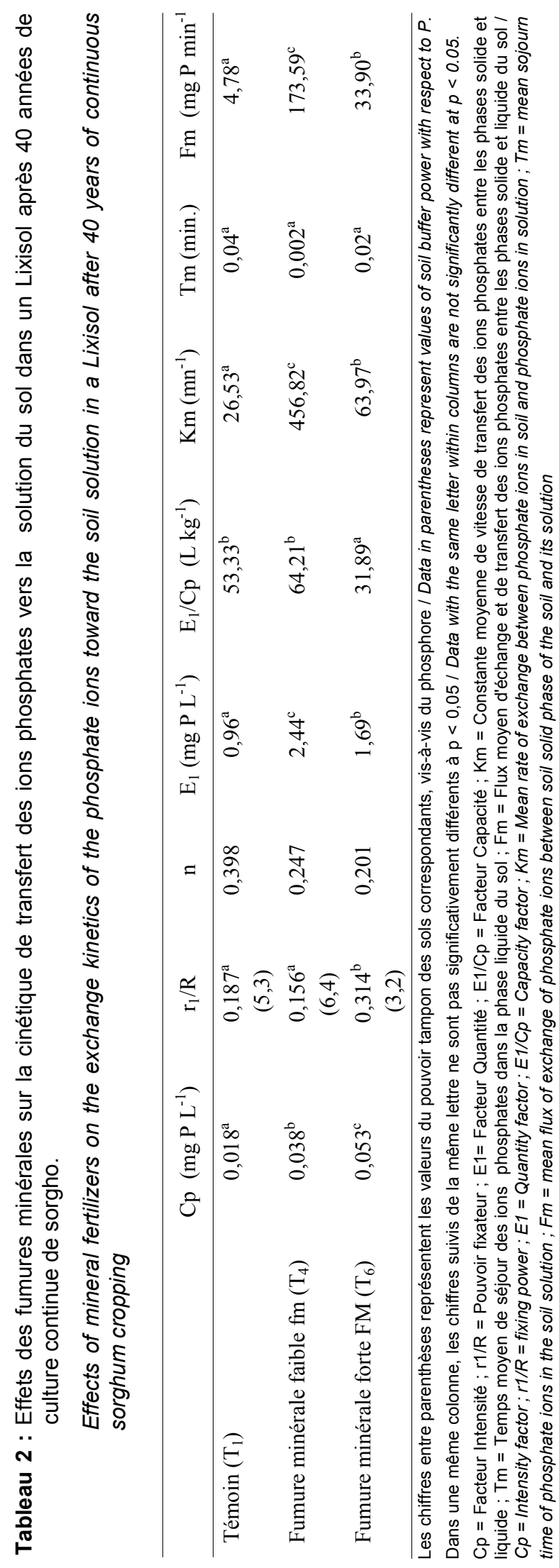




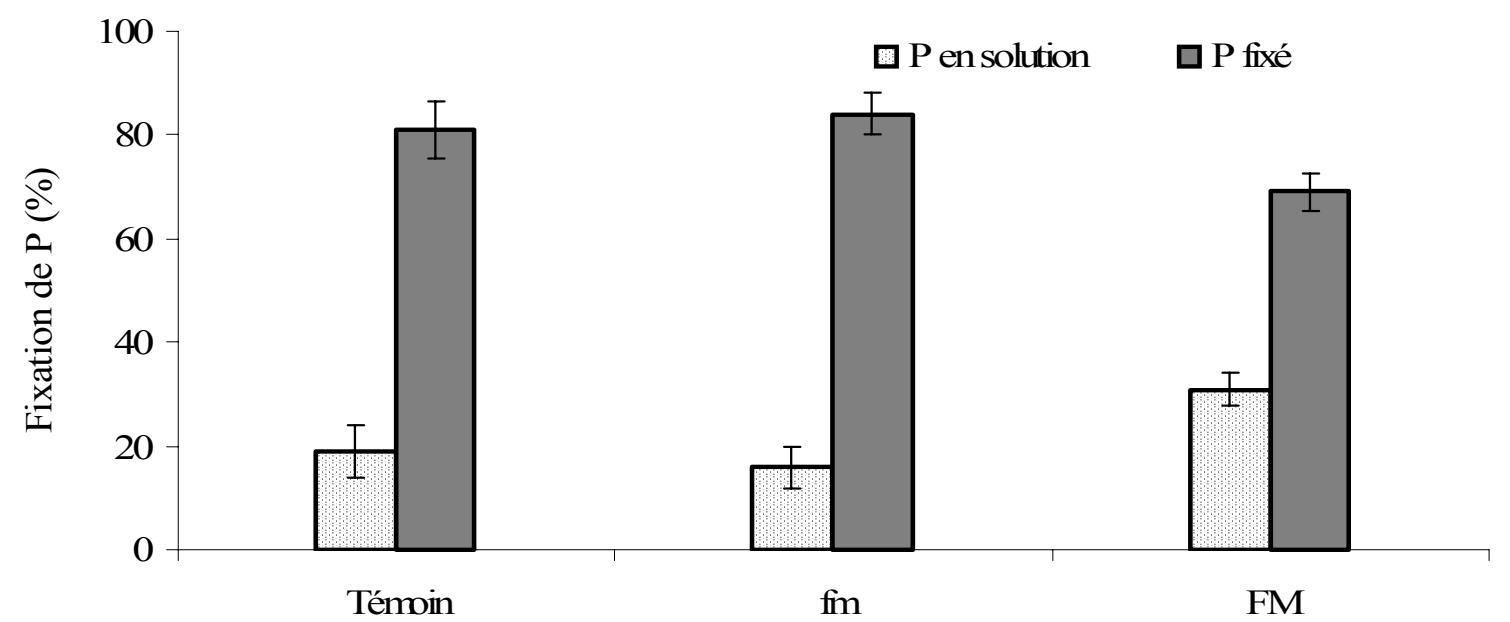

Figure 1 : Effets des fumures minérales sur la fixation du phosphore dans un Lixisol après 40 années de culture continue de sorgho.

Effect of mineral fertilizer rates on $P$ fixation in a Lixisol after 40 years of continuous sorghum cropping.

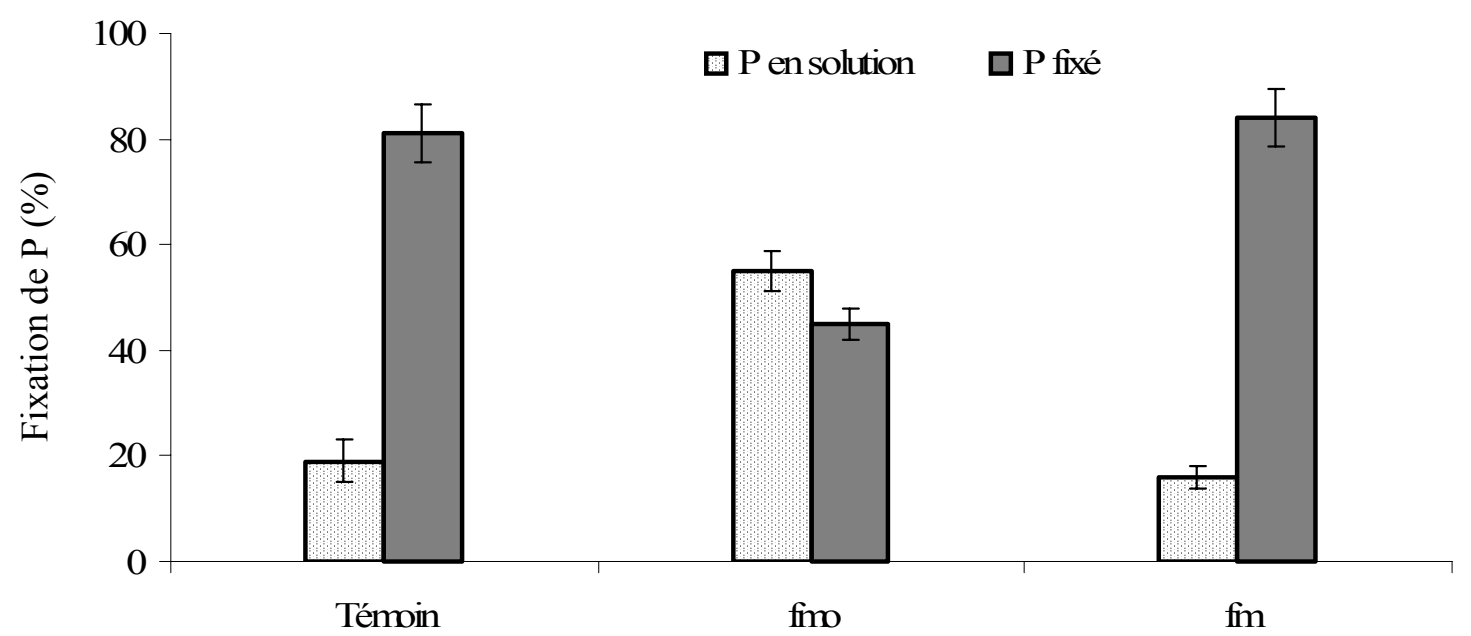

Figure 2 : Effets de la fumure organo-minérale sur la fixation du phosphore dans un Lixisol après 40 années de culture continue de sorgho.

Effect of the combination of organic input and mineral fertilizer on $P$ fixation in a Lixisol after 40 years of continuous sorghum cropping. 


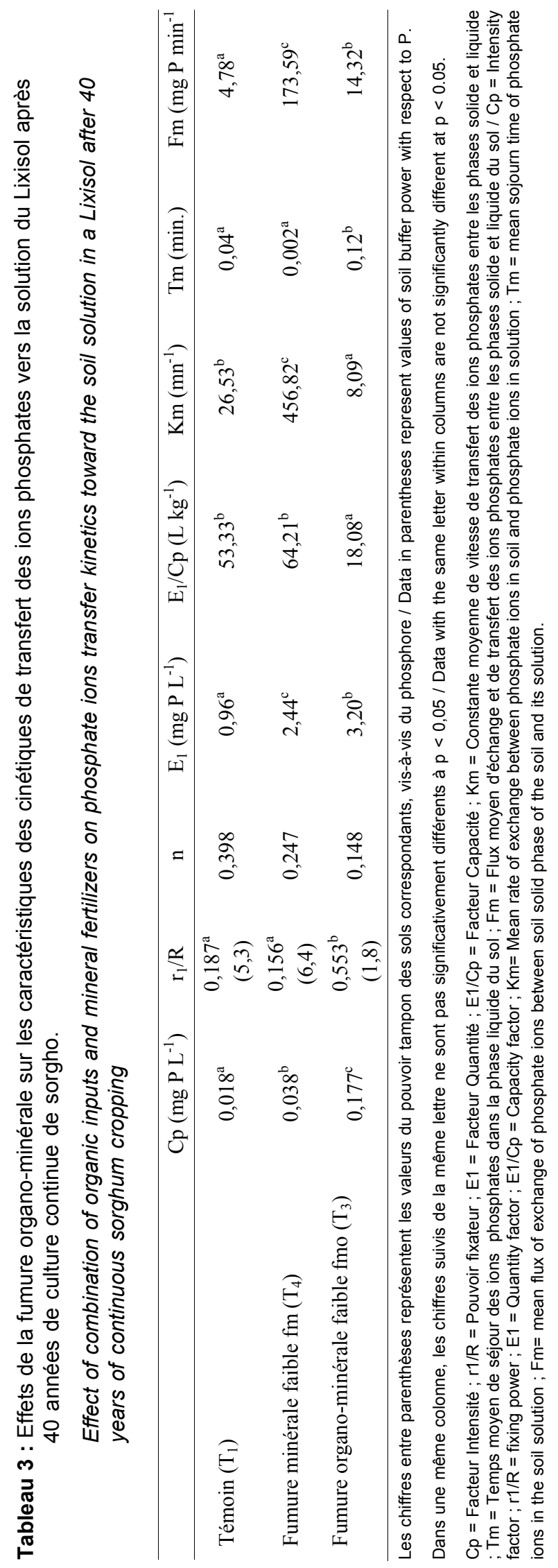




\section{Constantes moyennes de vitesse d'échange du phosphore}

La fumure organo- minérale a permis de réduire de façon significative $\mathrm{Km}$, comparativement aux fumures minérales (Tableau 3). Le flux moyen de transfert des ions (Fm) a connu la même évolution qu'avec la fumure $\mathrm{fm}$. Par contre, le temps moyen de séjour des ions phosphates dans la solution du sol avant tout renouvellement à partir d'échange avec les ions phosphates de la phase solide ( $\mathrm{Tm})$, a été plus long avec fmo qu'avec fm.

\section{CINETIQUES DE TRANSFERT DES IONS PHOSPHATES ET PROPRIETES PHYSICO- CHIMIQUESDU SOL}

Le facteur $\mathrm{Cp}$ a été positivement et significativement corrélé avec $\mathrm{P}$ assimilable, déterminé par les méthodes Bray $1\left(r^{2}=0,74\right)$ et Olsen $\left(r^{2}=0,76\right)$. Par contre, Cp a été négativement et significativement corrélé avec les limons fins $\left(r^{2}=-0,76\right)$. Les corrélations entre $\mathrm{Cp}$ et le $\mathrm{pH}$, d'une part, et les teneurs en argiles d'autres part, ont été négatives, mais faibles. Par contre, elles ont été positives, mais faibles, entre $\mathrm{Cp}$ et le carbone organique (Tableau 4).

Le $\mathrm{Cp}$, la CEC et les teneurs en $\mathrm{Ca}^{2+}$ ont varié en sens inverse, alors que $\mathrm{Cp}$ et $\mathrm{Mg}^{2+}$ ont varié dans le même sens. Ces observations confirment celles de Perrot (2003) qui ont montré que les teneurs élevées de $\mathrm{Ca}^{2+}$ dans la solution du sol diminuent celles de $\mathrm{P}$, alors que des teneurs élevées en $\mathrm{Mg}^{2+}$ ' $\mathrm{s}$ accompagnent de teneurs élevées de $P$.

Le facteur Quantité $\left(E_{1}\right)$ a été positivement et significativement corrélé avec $P$ Bray 1 $\left(r^{2}=0,86\right)$, et $P$ Olsen $\left(r^{2}=0,91\right)$. Ce facteur $a$ été positivement, mais faiblement corrélé avec le carbone organique du sol $\left(r^{2}=0,41\right)$. Par contre, les corrélations entre $\mathrm{E}_{1}$ et le $\mathrm{pH}$, les teneurs en argiles et en limons fins ont été négatives et non significatives.

Le pouvoir fixateur $\left(r_{1} / R\right)$ a été négativement et significativement corrélé avec les limons fins $\left(r_{2}=0,88\right)$. Les corrélations avec le $\mathrm{pH}$ et les teneurs en argiles ont été négatives et faibles. Le rapport $r_{1} / R$ a été positivement et significativement corrélé avec les sables fins $\left(r^{2}=0,83\right)$. 


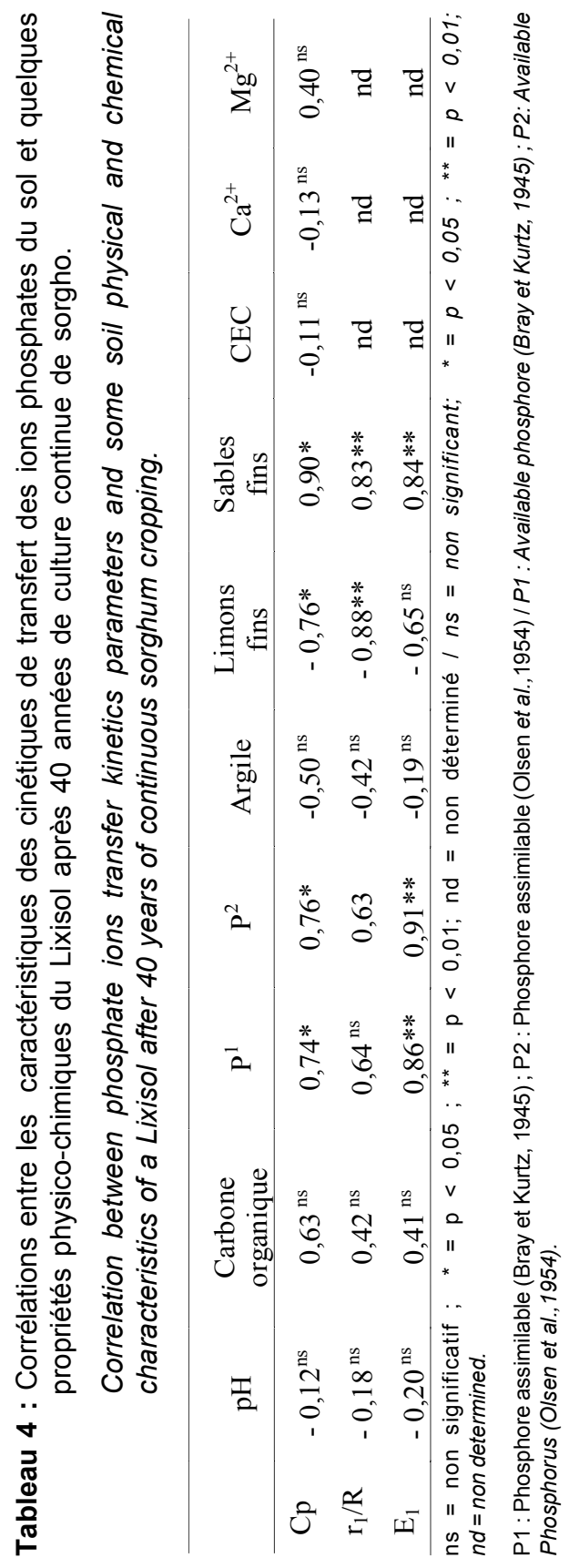

\section{DISCUSSION}

La fertilisation minérale a permis d'améliorer la disponibilité du phosphore dans un Lixisol. La dose faible (fm) a augmenté le plus le facteur Quantité, bien que la forte dose de NPK (FM) ait permis aussi d'augmenter ce paramètre comparativement au témoin. Les valeurs de $E_{1}$ obtenues, ont été toutes inférieures à $2,5 \mathrm{mg} \mathrm{P}$ $\mathrm{kg}^{-1}$, indiquant que cet élément demeure un facteur limitant pour la production des plantes (Sedogo et al.,1983 ; Lompo, 1993 ; Lompo et

al., 1994 ; Fardeau et al., 1996 ; Bado et al., 1997). Ceci confirme de nombreux travaux antérieurs menés au Burkina Faso sur ce sujet.

On peut cependant noter que la fertilisation minérale, avec des valeurs de $\mathrm{Cp}>0,02 \mathrm{mg} P$ $\mathrm{L}^{-1}$ peut permettre de satisfaire les besoins en $P$ des cultures (Fardeau et al., 1996 ; Compaoré et al., 2003). Les faibles valeurs de $\mathrm{Cp}$ et de $\mathrm{E}_{1}$ observées sont fortement liées au pouvoir tampon $\left(R / r_{1}\right)$ des sols vis-à-vis des apports extérieurs de $P$. Les valeurs de $R / r_{1}>5$, correspondent à 
des sols ayant un pouvoir tampon élevé avec une capacité maximale de rétention de $\mathrm{P}$. Ce sont généralement des sols dont l'argile dominante est la kaolinite à laquelle sont associés des oxydes métalliques, responsables de l'adsorption de P (Frossard, 1985 ; Fardeau, 1996). II existe en effet une forte corrélation entre $\mathrm{R} / \mathrm{r}_{1}$ d'une part et les teneurs en $\mathrm{Fe}, \mathrm{Al}$ et en argile d'autre part (Compaoré, 1996 ; Frossard et Sinaj, 1997).

Dans le sol témoin, qui présente un bilan en $P$ négatif (Lompo et al., 2008), il est probable que la nutrition phosphatée des plantes se fasse à partir du pool $E>1$ an. De même, la minéralisation du phosphore organique (Oehl et al., 1998) et l'altération du matériau parental (Letkeman et al.,1996), peuvent aussi contribuer à la nutrition phosphatée des plantes. Newman (1995) a estimé que la contribution de l'altération des roches à la nutrition phosphatée pouvait varier entre 0,05 et $5 \mathrm{~kg} \mathrm{P} \cdot \mathrm{ha}^{-1} \cdot \mathrm{an}^{-1}$. Ces différents processus (minéralisation de $P$ organique et altération des roches) peuvent concerner aussi bien fm et FM que $T_{1}$.

La fumure organo-minérale a eu des effets positifs aussi bien sur les formes que sur les fractions et le bilan de $\mathrm{P}$ (Lompo et al., 2008). Ces effets positifs (comparativement à fm) peuvent s'expliquer par l'accumulation de $\mathrm{P}$ organique du fait de la fumure organo-minérale (Sadler and Stewart, 1975). Le phosphore organique est minéralisé sous l'action des microorganismes et redistribué dans les différentes fractions du sol (Frossard, 1985) tout en contribuant à l'alimentation phosphatée des plantes (Chater and Mattingly, 1980 ; Hedley et al., 1982). L'effet bénéfique de la fumure organominérale sur le $\mathrm{P}$ assimilable est confirmé par les valeurs de $C p$ et de $E_{1}$ plus élevées.

Les hausses de $C p$ et de $E_{1}$, s'accompagnent d'une baisse du pouvoir fixateur du sol vis-à-vis de $P$. Oehl et al. (2002), ont trouvé des résultats similaires dans l'étude de la disponibilité de $P$ dans des sols en agriculture organique. La plus grande biodisponibilité du phosphore obtenue avec la fmo peut s'expliquer par (i) la production, au cours de la minéralisation de la matière organique apportée, d'anions organiques qui par complexation avec le $\mathrm{Fe}$, $\mathrm{Al}$ et $\mathrm{Ca}$, conduisent à une libération de $\mathrm{P}$ fixé (Pichot et al. 1973 ; Frossard, 1985 ; Garapin, 1989), et (ii) la compétition pour les sites de fixation du phosphore situés sur les particules de sol, entre les charges électronégatives issues de la minéralisation du fumier et les ions phosphates. Cette compétition aboutit à la fixation des ions sur les sites de plus faible énergie, les rendant ainsi plus labiles (Nagarajah et al., 1970, Earl et al., 1979 ; Samaké, 1987).

Le corollaire du pouvoir fixateur de $\mathrm{P}$ est le pouvoir tampon que Frossard et al. (1992) ont défini comme étant l'aptitude du phosphore du sol à maintenir $\mathrm{Cp}$ constant, quand $\mathrm{E}_{1}$ varie. Selon la classification proposée par Fardeau (1981), un sol a un pouvoir tampon élevé quand $R / r_{1}>5$; il est faible quand $R / r_{1}<2,5$. Partant de ces seuils, et des données sur les valeurs $R / r_{\text {, }}$ obtenues dans cette étude avec la fumure minérale faible, les sols ont un pouvoir tampon élevé, tandis que la fumure minérale forte leur confère un pouvoir tampon modéré. Ce pouvoir est faible avec la fumure organique. Ces résultats peuvent s'expliquer par la compétition entre la matière organique et le $\mathrm{P}$ pour les sites de sorption. II y'aurait donc moins de sites vacants avec la fumure organique et la fumure minérale forte.

Les variations du paramètre $\mathrm{n}$ confirment la capacité de ces sols à fixer $\mathrm{P}$ (Compaoré, 1996).

La constante moyenne de vitesse de transfert des ions entre les phases solide et liquide $(\mathrm{Km})$ a été significativement plus élevée avec la fumure minérale faible (traitement ayant le plus grand pourcentage de $\mathrm{P}$ fixé) et le temps moyen de séjour de $\mathrm{PO}_{4}^{3-}$ dans la phase liquide du sol (Tm), a été significativement plus court. Par contre, avec l'apport de matière organique (traitement ayant le plus grand pourcentage de $P$ en solution), $\mathrm{Km}$ a été plus faible et Tm plus élevé. Ces résultats montrent que les ions $\mathrm{PO}_{4}{ }^{3-}$ séjournent plus longtemps dans la solution du sol si celle-ci a une teneur élevée en P (Xiong et al., 2002).

Les valeurs des différentes constantes moyennes de transfert des ions phosphates de la phase solide vers la solution du sol $(\mathrm{Km}, \mathrm{Tm}$ et $\mathrm{Fm}$ ) sont dans le même ordre de grandeurs que celles obtenues sur d'autres sols tropicaux (Fardeau et al.,1996 ; Compaoré, 1996). La nutrition phosphatée du sorgho semble être satisfaisante avec les deux niveaux de fumures minérales dans la mesure où les valeurs de $\mathrm{Fm}$ obtenues sont supérieures à 30 que Fardeau (1981) considère comme le seuil en dessous duquel cette nutrition n'est plus satisfaisante. 


\section{CONCLUSION}

Quarante ans de fertilisations minérales ont eu un effet significatif sur la cinétique de transfert et les constantes de vitesse d'échange de $P$ dans un Lixisol. Comparativement au témoin, les Facteurs Intensité (Cp), et Quantité $\left(E_{1}\right)$ ont été améliorés par les différentes fumures respectivement de 153 et $115 \%$ en moyenne. Le Facteur Capacité $\left(E_{1} / C p\right)$, relativement constant lorsque l'on compare les fumures minérales et le témoin, a par contre, varié selon le niveau de fumure minéral (faible ou fort). La constante moyenne de vitesse $(\mathrm{Km})$ et le flux moyen (Fm) de transfert des ions phosphates entre les phases solide et liquide du sol ont été modifiés par les fumures minérales. Par contre les temps moyens de séjour des ions dans la phase liquide ( $\mathrm{Tm}$ ) ont été courts et n'ont pas semblé être influencés par les fumures minérales. Comparativement à la fertilisation minérale faible seule, la fumure organo-minérale, à faible dose a permis d'améliorer $\mathrm{Cp}$ et $\mathrm{E}_{1}$ de 366 et $31 \%$, respectivement. Par contre, $E_{1} / C p$ et le pouvoir fixateur ont baissé. Les temps moyens de séjour des ions phosphates dans la phase liquide ont été plus longs.

La fertilisation organo-minérale vulgarisée au Burkina Faso pour le sorgho (100 kg ha-1 $\mathrm{an}^{-1}$ du complexe NPK + $50 \mathrm{~kg} \mathrm{ha}^{-1} \mathrm{an}^{-1} \mathrm{~d}^{\prime}$ urée $+5 \mathrm{t}$ de fumier ha ${ }^{-1}$ tous les 2 ans) peut donc permettre d'améliorer la nutrition en $\mathrm{N}$ et $\mathrm{K}$ des plantes, et de satisfaire leurs besoins en $\mathrm{P}$.

\section{REFERENCES}

Bado B. V., M. P. Sedogo et F. Lompo. 1997. Efficacité d'un phosphatage de fond sur la productivité d'un sol ferrallitique. Soil Fertility Management in West Africa. Land Use Systems : $85-88$

Bationo A., W. E Baethgen, C. B. Christianson and A. U. Mokwunye. 1991. Comparison of five soil testing methods to establish phosphorus sufficiency levels in soil fertilized with water-soluble and sparingly soluble-P sources. Fert. Res. 28 : $271-279$

Beckett P. H. T. and R. E. White. 1964. Studies on the phosphate potential of soils. III. The pool of labile inorganic phosphate. Plant and Soil. 21: 253 - 282.

Boniface R. et S. Trocmé. 1988. Enseignements fournis par des essais de longue durée sur la fumure phosphate et potassique. 2 Essais sur la fumure phosphatée. In : L. Gachon (Eds.). Phosphore et potassium dans les relations sol-plante : conséquences sur la fertilisation. INRA, Paris : pp 279 - 402.

Bray R. H. and L. T. Kurtz. 1945. Determination of total, organic, and available forms of phosphorus in soils. Soil Science. 59 : 39-45.

Calvet R. 1988. Analyse du concept de biodisponibilité d'une substance dans le sol. Sci. Sol 2 : 183 - 202.

Chater M. and G. E. G. Mattingly. 1980. Changes in organic content of soils from long-continued experiments at Rothamsted and Saxmundham. Rothamsted Experimental Station Report for 1979. Part $2: 41$ - 61 .

Compaoré E. 1996. Contribution à la caractérisation et à la gestion de la fertilité phosphate de quelques sols ferrugineux tropicaux. Thèse de Doctorat, Université de Nancy (France), $147 \mathrm{p}$.

Compaoré E., E. Frossard, S. Sinaj, J. C. Fardeau and J. L. Morel. 2003. Influence of land-use management on isotopically exchangeable phosphate in soils from Burkina Faso. Commun. Soil Sci. Plant Anal. 34 (1 \& 2) : $201-223$

Di H. J., L. M. Condron and E. Frossard. 1997. Isotope techniques to study phosphorus cycling in agricultural and forest soils: A review. Biol. Fertil. Soils $24: 1$ - 12.

Earl K. D., J. K. Syers and J. R. McLaughlin. 1979. Origin of the effects of Citrate, tartrate and acetate on phosphate sorption by soils and synthetic gels. Soils Sci. Soc .Am. J. 43 : $674-678$.

Fallavier P., D. Babre et M. Bresse. 1985. Détermination de la capacité d'échange cationique des sols tropicaux. Agron. Trop. $40(4)$ : 298 - 308.

Fardeau J. C. and J. Jappe. 1980. Choix de la fertilisation phosphorique dans les sols tropicaux : emploi du phosphore $32, \mathrm{XXXV}$ : $225-231$

Fardeau J. C. 1981. Cinétiques de dilution isotopique et phosphore assimilable des sols. Thèse de Doctorat d'Etat ès Sciences. Option : Sol et Fertilisants, Université Pierre et Marie Curie, Paris 6, 197 p.

Fardeau J. C., C. Morel and J. Jappé. 1985. Cinétique d'échange des ions phosphate dans les systèmes sol : solution. Vérification expérimentale de l'équation théorique. C. 
R. Séances Acad. Sci. Paris, t. 300, III (8) : 371 - 376.

Fardeau J. C., C. Morel et R. Boniface. 1991. Cinétiques de transfert des ions phosphates du sol vers la solution du sol: paramètres caractéristiques. Agronomie. $11: 787$ - 797.

Fardeau J. C. 1996. Dynamics of phosphate in soils. An isotopic outlook. Fert. Res. 45 : $91-100$.

Fardeau J. C., G. Guiraud and C. Morel. 1996. The role of isotopic techniques on the evaluation of the agronomic effectiveness of $P$ fertilizers. Fert. Res. 45 : 101 - 109.

Fontes J. et S. Guinko. 1995. Carte de la végétation et de l'occupation du sol du Burkina Faso. Notice explicative. Projet Campus, ICIV, Toulouse, IDR-FAST, Ouagadougou, $57 \mathrm{p}$.

Frossard E. 1985. Etude expérimentale de l'influence de composés organiques sur l'évolution des ions phosphates en sol ferrallitique. Thèse de Doctorat, Université de Nancy (France), 109 p.

Frossard E., J. C. Fardeau, M. Ognalaga and J. L. Morel. 1992. Influences of agricultural practices, soil properties and parent material on the phosphate buffering capacity of cultivated developed in temperate climates. Eur. J. Agron. $1: 45$ - 50.

Frossard E. and S. Sinaj. 1997. The isotope exchange kinetic technique : a method to describe the availability of inorganic nutrients. Application to K, P, S and $\mathrm{Zn}$. Isotopes Environ. Health Stud. 33 : 61 - 77.

Garapin G. 1989. Influence des molécules organiques et des matières humiques sur la solubilisation d'une hydroxyapatite. Thèse de Doctorat $3^{\text {è }}$ cycle, Production et traitement des matières minérales, Université de Toulouse (France), $118 \mathrm{p}$.

Hedley M. J., J. W. B. Stewart and B. S. Chauhan. 1982. Changes in inorganic and organic soil phosphorus fractions induced by cultivation practices and by laboratory incubations. Soil Sci. Soc. Am. J. 46 : $970-976$.

luss, Isric, FAO, 2006. World reference base for soil resources 2006. A framework for international classification, correlation and communication ; $145 \mathrm{p}$.

Letkeman L. P., H. Tiessin and C. A. Campbell. 1996. Phosphorus transformations and redistribution during pedogenesis of western Canadian soil. Geoderma. 71 : $201-218$.
Lompo F. 1993. Contribution à la valorisation des phosphates naturels du Burkina Faso: Etude des effets de l'interaction phosphates naturels-matières organiques. Thèse de Docteur-Ingénieur, Sciences agronomiques, Université d'AbidjanCocody (Côte d'Ivoire), 249 p.

Lompo F., M. P. Sedogo et A. Assa. 1994. Effets à long terme des phosphates naturels de Kodjari (Burkina Faso) sur la production du sorgho et les bilans minéraux. Rev. Res. Amélior. Agr. Milieu Aride. 6 : 163 - 178.

Lompo F, M. Bonzi, B. V. Bado, Z. Gnankambary, N. Ouandaogo, P. M. Sedego et A. Assa. 2008. Influence à long terme des modes de gestion de la fertilité sur les états, les formes, les fractions et le bilan du phosphore d'un Lixisol du Burkina en culture continue de sorgho. Int. J. Biol. Chem. Sci. 2(2) : 175 - 184

Nagarajah S., A. M. Posner and J. P. Quirk. 1970. Competitive adsorption of phosphate with polygalacturonate and other organic anions on kaolinite and oxide surfaces. Nature / London 228 : 83 - 84.

Newman E. T. 1995. Phosphorus inputs to terrestrial ecosystems. J. Ecol. 83 : 713 - 726.

Oehl F., A. Oberson, E. Frossard, A. Fliessbach and M. Probst. 1998. Phosphorus in soil microbial biomass - Influence of conventional and biological farming. Proceedings of $16^{\text {th }}$ World Congress of soil science, 20 - 16 August 1998, Montpellier, France. Symp. 13B.

Oehl F., A. Oberson, H. U. Tagmann, J. M. Besson, D. Dubois, P. Mâder, H. R. Roth and E. Frossard. 2002. Phosphorus budget and phosphorus availability in soils under organic and conventional farming. Nutr. cycl. Agroecosyst. $62: 25$ - 35.

Olsen S. R., C. V. Cole, F. S. Watanabe, L. A. Dean. 1954. Estimation of available phosphorus in soils by extraction with sodium bicarbonate. USDA Circular $939: 8 \mathrm{p}$.

Perrot K. 2003. Direct application of phosphate rocks to pastoral soils-phosphate rock reactivity and influence of soil and climatic factors. In : S. S. S. Rajan and S. H. Chien (Eds.). Direct application of phosphate rock and related technology : latest developments and practical experiences. Proc. Int. Meeting, Kuala Lumpur, 16 - 20 July 2001 ; IFDC, Muscle Shoals, USA, $441 \mathrm{p}$.

Pichot J., S. Burdin et B. Truong. 1973. Evolution du phosphore dans un sol ferrallitique soumis à différents traitements agronomiques. Agron. Trop. 28 (2) : 131 - 139. 
Sadler J. M. and J. W. B. Stewart. 1975. Changes with time in form and availability of residual fertilizer phosphorus in a catenary sequence of Chernozemic. Soils. J. Soil Sci. 30 : $473-483$.

Samaké F. 1987. Contribution à la valorisation du phosphate naturel de Tilemsi (Mali) par l'action d'acides minéraux et de composés organiques humifiés. Thèse de Docteur Ingénieur. Université de Nancy (France), $198 \mathrm{p}$.

Sedogo P. M., I. M. Bikienga et D. Ouattara. 1983. Utilisation agricole des phosphates naturels de Haute-Volta. Synthèse des premiers résultats ; Notes et Documents voltaïques 14 (2) : 30 - 39.
Sinaj S., A. Buerkert, G. El-Hajj, A. Bationo and H. Traoré. 2001. Effect of fertility management strategies on phosphorus bioavailability in four West African soils. Plant Soil. 233 : $71-83$.

Walinga I., W. Vark, V. J. G. Houba and J. J. Van Der Lee. 1989. Soil and plant analysis, Part 7. Department of Soil Science and Plant Nutrition, Wageningen Agricultural University, Wageningen, The Netherlands.

Walkley A. and A. Black. 1934. An examination of the Degtjareff method for determining soil organic matter and a proposed modification of the chromic acid titration method. Soil Sci. $37: 29$ - 38 